\title{
Identification and characterization of microRNAs related to salt stress in broccoli, using high-throughput sequencing and bioinformatics analysis
}

Yunhong Tian ${ }^{1,2}$, Yunming Tian ${ }^{3,4}$, Xiaojun Luo', Tao Zhou², Zuoping Huang ${ }^{2}$, Ying Liu², Yihan Qiu², Bing Hou², Dan Sun'², Hongyu Deng ${ }^{1}$, Shen Qian² and Kaitai Yao ${ }^{1 *}$

\begin{abstract}
Background: MicroRNAs (miRNAs) are a new class of endogenous regulators of a broad range of physiological processes, which act by regulating gene expression post-transcriptionally. The brassica vegetable, broccoli (Brassica oleracea var. italica), is very popular with a wide range of consumers, but environmental stresses such as salinity are a problem worldwide in restricting its growth and yield. Little is known about the role of miRNAs in the response of broccoli to salt stress. In this study, broccoli subjected to salt stress and broccoli grown under control conditions were analyzed by high-throughput sequencing. Differential miRNA expression was confirmed by real-time reverse transcription polymerase chain reaction (RT-PCR). The prediction of miRNA targets was undertaken using the Kyoto Encyclopedia of Genes and Genomes (KEGG) Orthology (KO) database and Gene Ontology (GO)-enrichment analyses.
\end{abstract}

Results: Two libraries of small (or short) RNAs (sRNAs) were constructed and sequenced by high-throughput Solexa sequencing. A total of 24,511,963 and 21,034,728 clean reads, representing 9,861,236 (40.23\%) and 8,574,665 (40.76\%) unique reads, were obtained for control and salt-stressed broccoli, respectively. Furthermore, 42 putative known and 39 putative candidate miRNAs that were differentially expressed between control and salt-stressed broccoli were revealed by their read counts and confirmed by the use of stem-loop real-time RT-PCR. Amongst these, the putative conserved miRNAs, miR393 and miR855, and two putative candidate miRNAs, miR3 and miR34, were the most strongly down-regulated when broccoli was salt-stressed, whereas the putative conserved miRNA, miR396a, and the putative candidate miRNA, miR37, were the most up-regulated. Finally, analysis of the predicted gene targets of miRNAs using the GO and KO databases indicated that a range of metabolic and other cellular functions known to be associated with salt stress were up-regulated in broccoli treated with salt.

Conclusion: A comprehensive study of broccoli miRNA in relation to salt stress has been performed. We report significant data on the miRNA profile of broccoli that will underpin further studies on stress responses in broccoli and related species. The differential regulation of miRNAs between control and salt-stressed broccoli indicates that miRNAs play an integral role in the regulation of responses to salt stress.

Keywords: Broccoli, Salt stress, High-throughput sequencing, microRNA

\footnotetext{
*Correspondence: yao.kaitai@yahoo.com.cn

${ }^{1}$ Cancer Research Institute, Southern Medical University, Guangzhou,

Guangdong Province, People's Republic of China

Full list of author information is available at the end of the article
} 


\section{Background}

MicroRNAs (miRNAs) are a class of non-coding small RNAs (sRNAs), approximately 20-24 nucleotides in length, that post-transcriptionally regulate gene expression. In plants, highly conserved and species-specific miRNAs control a vast array of biological processes, such as leaf polarity, flower development and stress responses $[1,2]$. The miRNAs are excised from stem-loop structures within larger primary miRNA transcripts by Dicer-Like1 (DCL1), which in each case trims the hairpin structure (pre-miRNA). In plants, the mature miRNA strands have high complementarity (fewer than four mismatches) to their target mRNAs and regulate gene expression via mRNA cleavage [3-5].

Analysis indicates that many plant miRNAs and their targets are evolutionarily conserved from species to species within the plant kingdom. Thus, a miRNA in one species may exist as orthologs or homologs in other species [6,7]. Plant miRNAs have usually been identified either by prediction through bioinformatics or by experimental methods [8]. However, the bioinformatics-based approaches can only identify miRNAs that are conserved amongst organisms, and DNA or RNA sequence information is required in order to run the programs. Thus, sequencing is the most effective method for plant miRNA discovery [9]. To date, 7384 sequences of mature miRNAs have been identified in plants, including 337 from $A$. thaliana, 384 from A. lyrata, 713 from O. sativa and 43 from B. rapa (miRBase release 20.0, June, 2013, http:// mirbase.org/).

The genus Brassica is cultivated in most parts of the world. It includes various crops of agronomic importance, such as broccoli, cauliflower and B. rapa. Broccoli (Brassica oleracea var. italica) is very popular with a wide range of consumers because of its flavor, and also on account of its anti-cancer activities, notably in relation to prostate and colorectal cancers [10]. Because broccoli, A. thaliana and B. rapa all belong to the same family, the Cruciferae, the level of synteny between these species provides a basis for studying the miRNAs of broccoli $[11,12]$.

Environmental stresses such as salinity are a worldwide problem in agriculture, diminishing plant growth and yield. Broccoli is moderately tolerant to salinity, and it displays better tolerance than some other common vegetables, such as maize and carrot [13,14]. Although several stress-related miRNAs have been identified based on the sequencing of a library of sRNAs isolated from A. thaliana seedlings, O. sativa, saccharum officinarum L. and from populous euphratica exposed to various stresses [15-19], there have been few studies that have focused upon miRNAs in broccoli, and in particular upon the identification of stress-related miRNAs in broccoli.
Thus, in the present study, we have identified miRNAs and their targets related to salt-stress in broccoli, using high-throughput sequencing methods. The differential expression of miRNAs observed between broccoli grown under standard conditions and broccoli subjected to salt stress provides new insights that will inform the genetic improvement of stress tolerance in plants.

\section{Results}

\section{Sequence analysis of sRNAs}

In order to identify the miRNAs responding to salt stress, we constructed and sequenced sRNA libraries ranging in size from 18 to $30 \mathrm{nt}$, from both control and salt-stressed broccoli. A total of 24,655,210 and 21,196,508 reads were obtained from control and salt-stressed broccoli, respectively. After removing the tags with: any $\mathrm{N}$ bases, more than 4 bases whose quality score was lower than 10 and more than 6 bases whose quality score was lower than 13 , and those that were too small (with length shorter than $18 \mathrm{nt}$ ), as well as the adapter sequences, 24,511,963 (99.74\%) and 21,034,728 (99.56\%) clean reads were obtained (Additional file 1: Table S1), representing 9,861,236 (40.23\%) and 8,574,665 (40.76\%) unique, although sometimes partially overlapping, clean reads from control and salt-stressed broccoli, respectively. The size distributions of the reads in the two datasets were quite similar, and they were not evenly distributed. The majority of these unique sequence reads were $24 \mathrm{nt}$ in size, followed by $23 \mathrm{nt}, 21 \mathrm{nt}$ and $22 \mathrm{nt}$, both in control and salt-stressed broccoli, which is similar to the size distribution of sRNAs in plants (Figure 1A). The overall distribution pattern of sRNAs (21 nt sRNAs $=16.81 \%$, and 24 nt sRNAs $=$ $52.01 \%$ ) in salt-stressed broccoli was similar to that in control broccoli $(21 \mathrm{nt}$ sRNAs $=15.23 \%$, and $24 \mathrm{nt}$ sRNAs $=$ $48.81 \%)$. Moreover, common and specific tags between the control and salt-stressed broccoli were analyzed. The results showed that only $2,419,170(15.10 \%)$ of the unique sequences and 29,295,190 (64.32\%) of the total sequences were shared between the two samples (Figure 1B), suggesting that the sequence results were reliably representative of the endogenous sRNAs in broccoli.

Because the whole genome of sequence for broccoli was not available, all clean reads were aligned against the $B$. rapa genome, using short oligonucleotide alignment program-2 (SOAP2, http://soap.genomics.org.cn) [20]. However, only a very small percentage of the unique sRNA sequences could be matched against the $B$. rapa genome; only $1,491,240(15.12 \%)$ of the reads in the control broccoli and 1,276,113 (14.88\%) of the reads in the salt-stressed broccoli could be mapped in this way, representing 7,163,496 (29.22\%) and 6,168,917 (29.33\%) of the total reads in control and salt-stressed broccoli, respectively (Additional file 2: Table S2). All clean reads were 

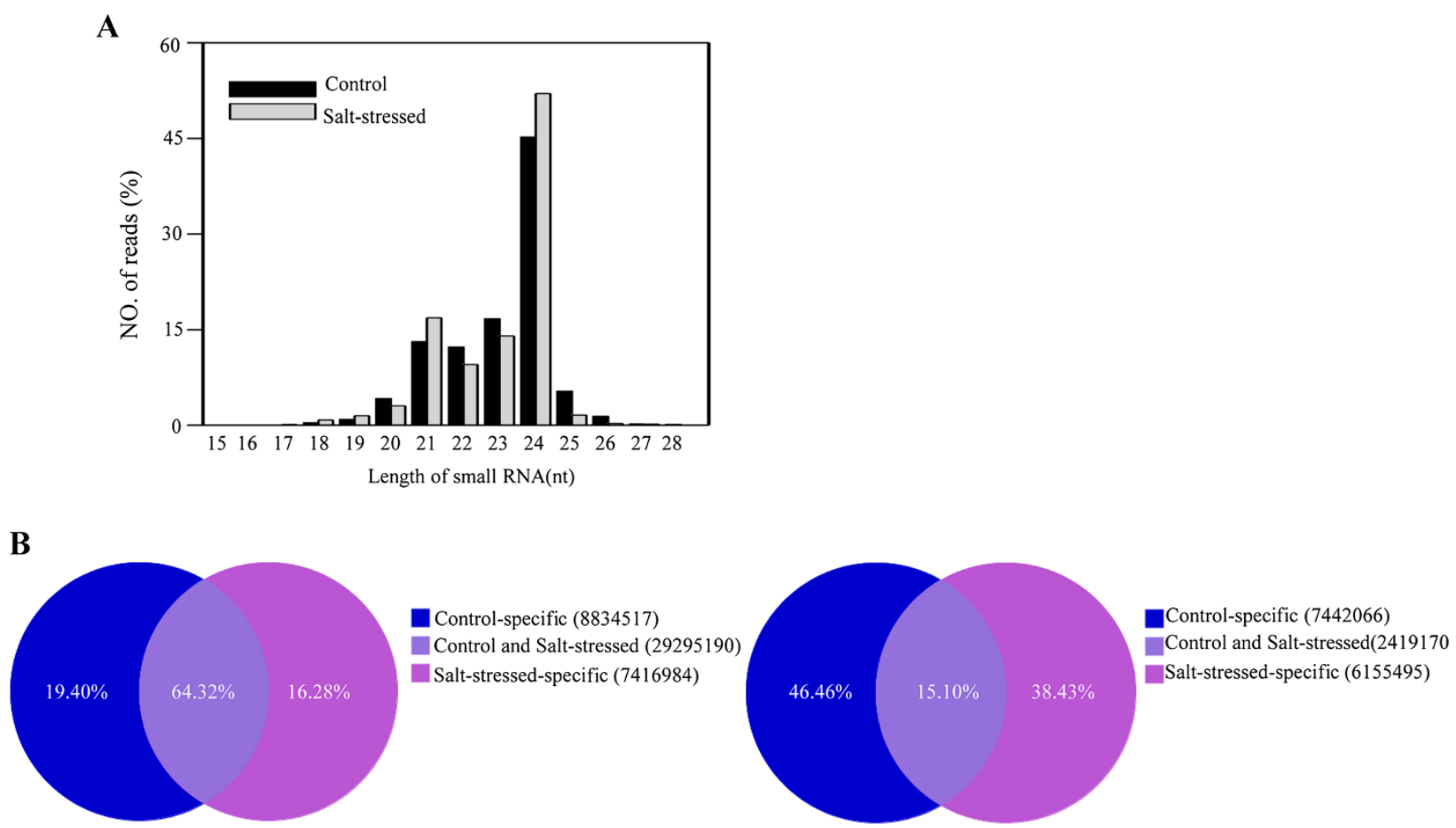

Figure 1 Sequence analysis of sRNAs. (A) Length distribution of sRNAs. The length distribution of high-quality sequences obtained from the two broccoli libraries. The distributions of the total reads are shown as percentages. (B) Summary of common and specific sequences between control and salt-stressed broccoli libraries. Total sRNAs and unique sRNAs are shown in the left panel and the right panel, respectively.

annotated into different categories, including plant miRNAs (miRbase, http://www.mirbase.org/), exons and introns (B. rapa genome, http://www.ncbi.nlm.nih.Gov/ genbank), and non-coding RNAs (Rfam, http://www. sanger.ac.uk). In those cases in which sRNAs were mapped to more than one category, the following priority rule was adopted: rRNA > known miRNA > exon > intron. The results indicated that the majority of sRNAs $-91.90 \%$ of the unique reads in the control group and $92.23 \%$ in the salt-stressed group - remained unannotated. For the control group, when unique sRNAs were matched, a small proportion of reads were derived from repeated sequences (3.98\%), and a smaller proportion from rRNAs (1.39\%). However, for the total sRNA pools, rRNAs $(7.79 \%)$ were the most abundant sequences, followed by repeated sequences (5.72\%) (Table 1; Additional file 3: Figure S1). All the unannotated sequences were then used for further analysis.

Table 1 Distribution of genome-mapped sequence reads in the sRNA libraries for control and salt-stressed broccoli

\begin{tabular}{|c|c|c|c|c|c|c|c|c|}
\hline \multirow[t]{2}{*}{ Locus class } & \multicolumn{4}{|l|}{ Control } & \multicolumn{4}{|l|}{ Salt-stressed } \\
\hline & Unique sRNA & Percent (\%) & Total sRNA & $\overline{\text { Percent (\%) }}$ & Unique sRNA & Percent (\%) & Total sRNA & Percent (\%) \\
\hline Total & 9861236 & $100 \%$ & 24511963 & $100 \%$ & 8574665 & $100 \%$ & 21034728 & $100 \%$ \\
\hline Exon antisense & 33857 & $0.34 \%$ & 91879 & $0.37 \%$ & 39594 & $0.46 \%$ & 83220 & $0.40 \%$ \\
\hline Exon sense & 56641 & $0.57 \%$ & 168950 & $0.69 \%$ & 61307 & $0.71 \%$ & 138900 & $0.66 \%$ \\
\hline Intron antisense & 60209 & $0.61 \%$ & 215558 & $0.88 \%$ & 53351 & $0.62 \%$ & 168011 & $0.80 \%$ \\
\hline Intron sense & 88950 & $0.90 \%$ & 406032 & $1.66 \%$ & 78075 & $0.91 \%$ & 331915 & $1.58 \%$ \\
\hline miRNA & 6595 & $0.07 \%$ & 810592 & $3.31 \%$ & 6385 & $0.07 \%$ & 1053521 & $5.01 \%$ \\
\hline rRNA & 137427 & $1.39 \%$ & 1909705 & $7.79 \%$ & 114451 & $1.33 \%$ & 1807910 & $8.59 \%$ \\
\hline repeat & 392888 & $3.98 \%$ & 1402050 & $5.72 \%$ & 295752 & $3.45 \%$ & 904109 & $4.30 \%$ \\
\hline snRNA & 4766 & $0.05 \%$ & 13349 & $0.05 \%$ & 5028 & $0.06 \%$ & 13861 & $0.07 \%$ \\
\hline snoRNA & 3159 & $0.03 \%$ & 8316 & $0.03 \%$ & 2250 & $0.03 \%$ & 4588 & $0.02 \%$ \\
\hline tRNA & 14221 & $0.14 \%$ & 300106 & $1.22 \%$ & 10021 & $0.12 \%$ & 192288 & $0.91 \%$ \\
\hline
\end{tabular}


Identification and expression patterns of salt-stressinduced conserved miRNAs in broccoli

Conserved miRNA families are found in many plant species and play an important role in a diversity of plant processes. In spite of this, sequence information for broccoli miRNAs is absent from both the miRBase database and from the plant miRNA database. Furthermore, although miRNA families are conserved between closely related species [6,21], there are only 43 known $B$. rapa miRNAs in miRBase. Therefore, the sRNA sequences were aligned to the miRNA precursor/mature miRNA sequences of the Viridiplantae in miRBase. Ninety-seven putative known miRNAs were identified in salt-stressed broccoli, including 72 miRNAs that were also found in the control group (Additional file 4: Table S3). In particular, the putative conserved miRNAs, miR156a, miR166a and miR168a, which have been found in about 40 plant species (miRBase release 20.0), were amongst the miRNAs that were found in both groups. A comparison of the miRNAs in the two libraries indicated that about 40 putative miRNAs - including, for example, miR393, miR841 and miR5658 - were less abundant in saltstressed broccoli than in control broccoli. Conversely, 45 miRNAs - including, for example, miR157d, miR393b$3 \mathrm{p}$ and miR169m - were more abundant in salt-stressed broccoli than in control broccoli (Figure 2A). Although the levels of some miRNAs belonging to the same miRNA gene family, such as miR157, miR158 and miR160, were found to be increased and decreased in parallel, others were not; the levels of miR393 and miR393a, for example, increased and decreased differently (Additional file 4: Table S3). However, when the fold-change $(\log 2)$ criterion between salt-stressed broccoli and control broccoli was set to $<-1.0$ or $>1.0$, and the false discovery rate (FDR) was set to $<0.01$, the levels of 45 miRNAs were found to be significantly different between salt-stressed and

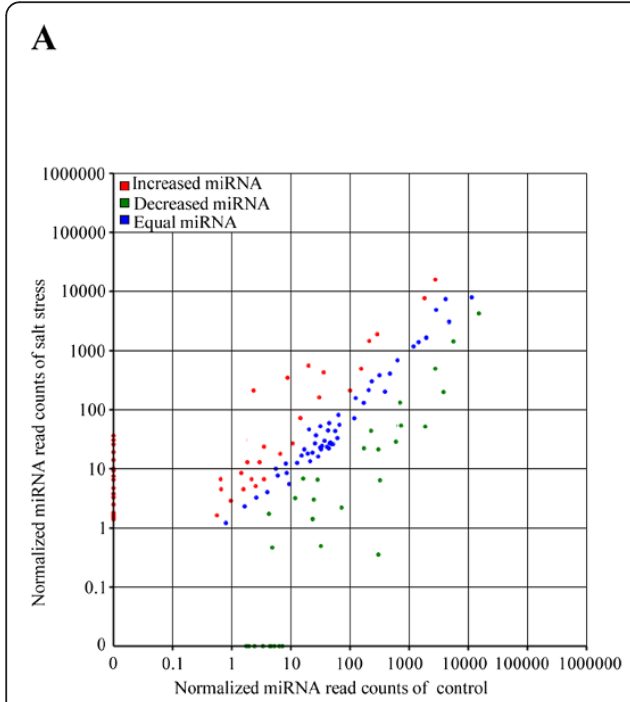

C

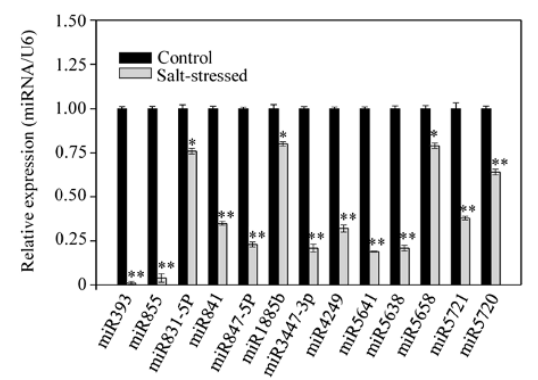

B

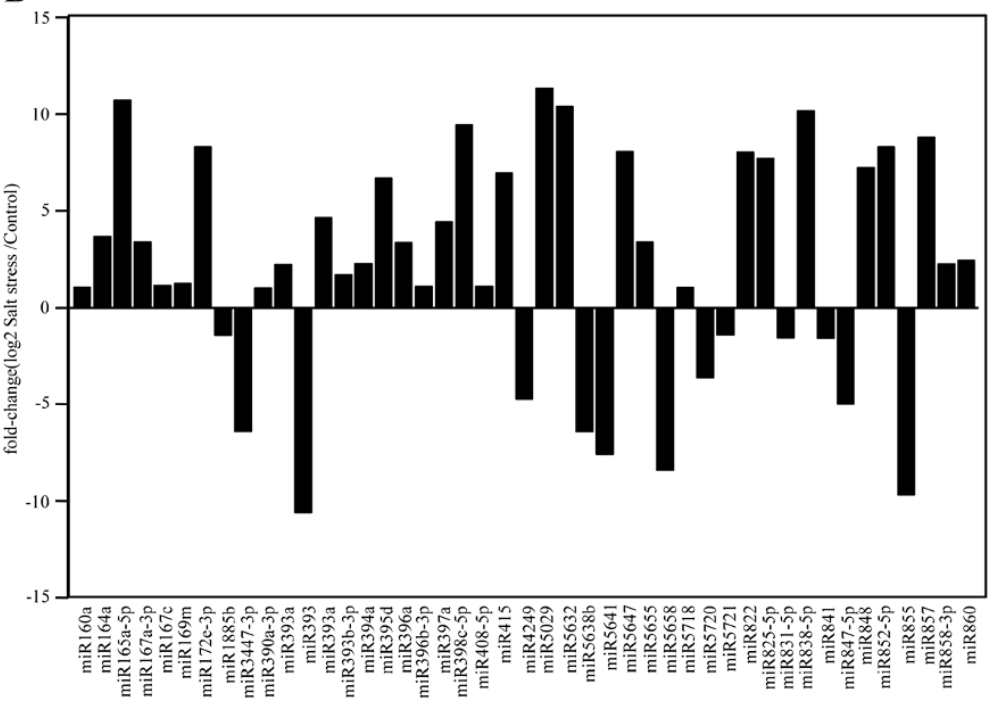

D

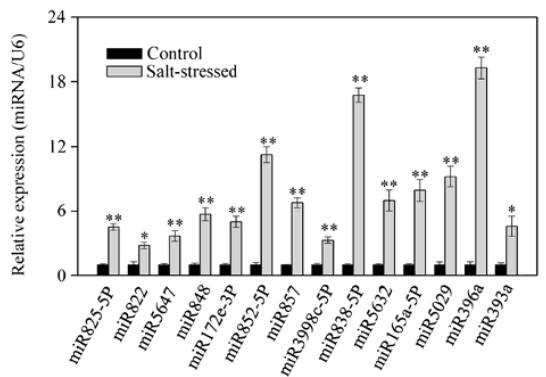

Figure 2 Differential expression of putative conserved miRNAs between control and salt-stressed broccoli. (A) Scatter diagram of the differential read counts of known miRNAs. Each point in the figure represents a miRNA. Red points represent miRNAs showing a $>2$-fold change of expression; blue points represent miRNAs showing $1 / 2<$ fold change $\leq 2$; green points represent miRNAs showing a fold change $\leq 1 / 2$. (B) The 45 miRNAs showing the greatest changes in expression, with fold changes $<1 / 2$ or $>2$. Decreased (C) and increased (D) putative conserved miRNAs confirmed by stem-loop real-time RT-PCR, as described in Methods. Significant difference between salt-stressed broccoli and control broccoli is indicated by ${ }^{*} P<0.05$ and ${ }^{*} P<0.01$. 
control plants. As shown in Figure 2B, the levels of miR393, miR855, miR841, miR168a and miR5641 were all decreased; levels of miR160, miR396a, miR397a and miR164a, on the other hand, were increased under salt stress. The miRNA that showed the greatest decrease under salt stress was miR393 and the miRNAs that showed the greatest increase were miR165a-5P and miR5029.

Real-time quantification of miRNAs by stem-loop realtime reverse transcription polymerase chain reaction (RT-PCR) is specific for mature miRNAs and discriminates amongst related miRNAs that differ by as little as one nucleotide. Furthermore, it is not affected by genomic DNA contamination [22,23]. Therefore, this technique was used to validate the sequencing results. To confirm the identity of the individual PCR products, they were firstly confirmed by electrophoretic sequencing and by denaturing gel electrophoresis. For those miRNAs that were less abundant in salt-stressed broccoli than in control broccoli, the results obtained by stem-loop RTPCR agreed with the results obtained by sequencing. The greatest degree of down-regulation in response to salt stress was shown by miR393 and miR855 (Figure 2C). Amongst the 32 miRNAs that were more abundant in salt-stressed broccoli than in control broccoli, 29 also showed up-regulated expression when analyzed by stemloop RT-PCR; the three exceptions were miR5655, miR858-3P and miR415, which were not detected by this technique, either in control or in salt-stressed broccoli. Of these 29, miR396a showed the greatest degree of up-regulation, followed by miR838-5P, miR852-5P, miR848 and miR393a (Figure 2D). These findings contrasted with the results obtained by sequencing, which indicated that the two most abundant up-regulated miRNAs were miR165a-5P and miR5029. In summary, 42 putative conserved miRNAs that were differentially expressed between control and salt-stressed broccoli were revealed by their read counts and confirmed by the use of stem-loop real-time RT-PCR.

\section{Identification and expression patterns of salt-stress- induced, non-conserved miRNAs in broccoli}

In order to identify putative novel miRNAs in the broccoli dataset, secondary structures and minimum freeenergies were calculated. In total, 326 unique mature miRNAs were identified initially in our study, and these candidate miRNAs originated from predicted RNA hairpins. With an average of about 46 read counts, these putative novel miRNAs showed fewer read counts than the putative conserved miRNAs. A comparison of the putative novel miRNAs in the two libraries indicated that the levels of about 59 of theses miRNAs were decreased in response to salt stress. Conversely, the levels of about 50 miRNAs were increased (Figure 3A). Real time RT-PCR was next performed for those miRNAs with an average of more than 46 read counts either in the salt stressed broccoli or control broccoli, and whose levels were found to be significantly different between salt-stressed and control plants, when the fold-change (log2) criterion between salt-stressed broccoli and control broccoli was set to $<-1.0$ or $>1.0$, and the FDR was set to $<0.01$. The results from the 52 miRNAs analyzed by real time RTPCR indicated that only 43 of these candidate miRNAs were expressed in either the control or the salt-stressed group (Table 2). A higher proportion of the sequences were identified from the 5'-ends of the hairpins than from the 3'-ends. Secondary hairpin structures for representative miRNAs are shown in Figure 3B, and the secondary hairpin structures for all the candidate miRNAs are listed in Additional file 5: Figure S2.

Some of the putative novel miRNAs showed particular expression profiles. Thus, eight were expressed only in libraries generated either from control or from saltstressed broccoli: three (miR5, miR8, miR24 and miR31) were detected only in control broccoli, whilst five (miR6, miR10, miR12, miR30 and miR39) were found only in salt-stressed broccoli. Four other putative novel miRNAs (miR2, miR9, miR16 and miR29) exhibited similar expression levels in both libraries. Although most of the putative novel miRNAs were observed in both libraries, their expression could be significantly different; for example, miR32 showed four-fold higher expression in salt-stressed broccoli than in control plants. Amongst those that were significantly up-regulated, miR37 was upregulated to the greatest extent, followed by miR1, miR14 and miR20 (Figure 3C). In contrast, miR3 and miR34 were the putative novel miRNAs that showed the largest downregulation in response to salt stress (Figure 3D). In summary, 39 new candidate miRNAs that were differentially expressed between control and salt-stressed broccoli were identified by their read counts and confirmed by the stemloop real-time RT-PCR.

\section{Target prediction and analysis of differential expression of miRNAs}

To understand the biological mechanisms by which broccoli responds to salt stress, the putative target sites of miRNAs were identified by aligning miRNA sequences with the Expressed Sequence Tags (ESTs) of B. rapa, following the rules of target prediction suggested by Allen et al. [24]. A total of 836 and 527 mRNAs were predicted as targets of putative conserved miRNAs and putative novel miRNAs, respectively. In plants, the identification of mRNA targets is straight forward because most miRNAs and their target mRNAs have exact or nearly exact complementarity. Furthermore, the miRNA target sites of plants have been shown to be located primarily in the coding regions [25]. 


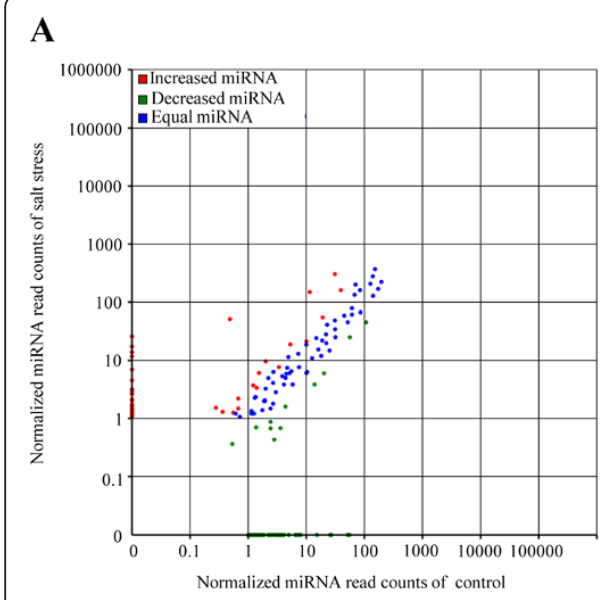

C

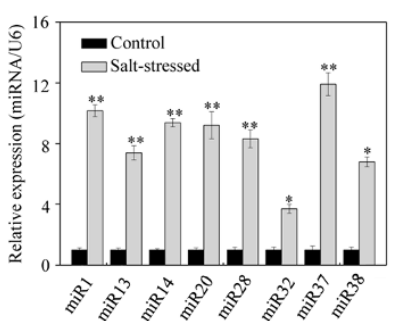

B

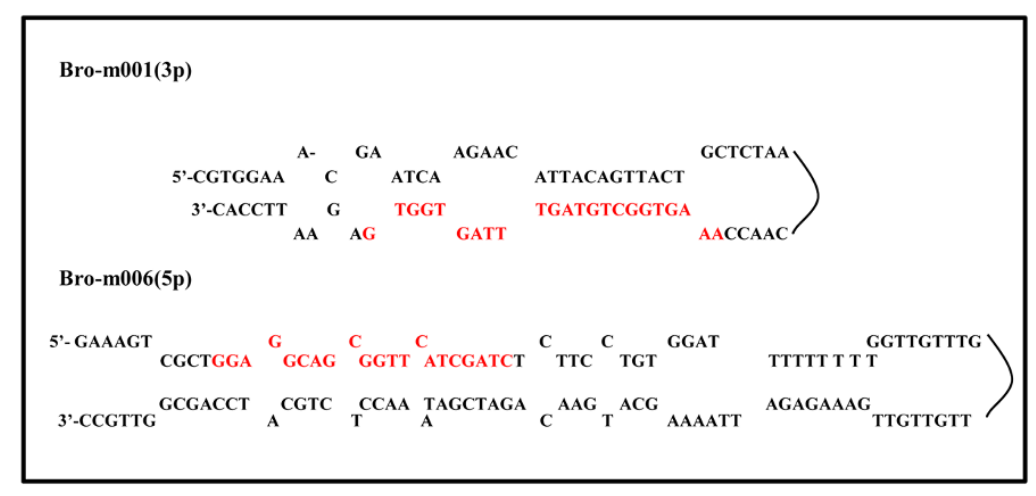

D

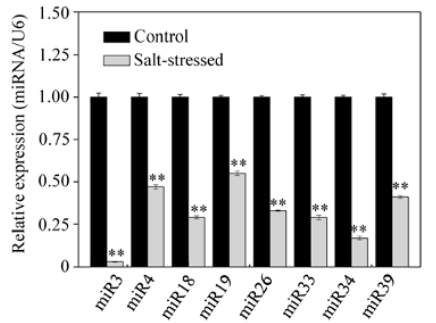

Figure 3 Differential expression of putative novel miRNAs between control and salt-stressed broccoli. (A) Scatter diagram of differential read counts of putative miRNAs. (B) Prediction of secondary structure of representative putative miRNAs from broccoli with or without salt treatment. Increased (C) and decreased (D) putative novel miRNAs confirmed by stem-loop real-time RT-PCR. Significant difference between salt-stressed broccoli and control broccoli is indicated by ${ }^{*} P<0.05$ and ${ }^{*} P<0.01$.

The putative target genes appeared to be involved in a wide variety of biological processes and moleculargenetic functions; therefore, the Gene Ontology (GO) database and the Kyoto Encyclopedia of Genes and Genomes (KEGG) Orthology (KO) database were used to analyze the datasets. Studies have shown that salt stress interferes with cell-cycle regulation at the transcriptional level, resulting in an adaptive growth response. Hormone signal transduction and sulfur metabolism are also vital for the improvement of stress tolerance in crop plants [26,27]. Interestingly, the results we obtained using the $\mathrm{KO}$ database indicated that genes involved in a diversity of cellular processes, such as the cell cycle, plant hormone signal transduction, and sulfur metabolism (Additional file 6: Table S4) were up-regulated in response to salt stress. Similarly, pathway annotation for the putative novel miRNAs that were up-regulated in response to salt stress indicated involvements in calcium reabsorption and in the cell cycle (Additional file 7: Table S5).

The gene targets of both the putative conserved and the putative novel miRNAs were classified into three GO categories: cellular location, biological process and molecular function. For the cellular location category, large numbers of targets for putative conserved and putative candidate miRNAs were categorized under 'cell' and 'cell part', whereas 'extracellular region' comprised the smallest proportion (Figure 4A and B). For the biological process category, the majority were classified under 'cellular process', 'metabolic process' or 'response to stimulus'. Under the molecular function category, the two most abundant sub-categories for both putative conserved and putative novel miRNAs were 'binding' and 'catalytic activity'.

\section{Discussion}

Salinity is an increasingly important agricultural problem. The metabolism and physiological integrity of plants are affected by salt stress and in recent years many studies have been devoted to understanding the molecular mechanisms of plant salt tolerance. Studies have confirmed that resistance to salt stress is associated with the use of compatible solutes by plants [28], and with ion transporters [29]. miRNAs are a class of non-coding sRNAs that are implicated in many developmental processes and in responses to various abiotic stresses, and which play pivotal roles in plant adaptation [1]. To date, in excess of 24,000 hairpin sequences and 30,000 mature sequences have been identified from plants, animals, unicellular organisms and viruses (miRBase); however, scant attention has been paid 
Table 2 Putative novel miRNAs in the two libraries

\begin{tabular}{|c|c|c|c|c|c|}
\hline Novel ID & Location & Strand $(+/-)$ & Energy (kcal/m & Sequence of $5 \mathrm{P}$ & Sequence of 3P \\
\hline Bro-m001 & A01:146563:146640 & + & -20 & - & AAAGTGGCTGTAGTTIAGTGG TG \\
\hline Bro-m002 & A01:13726926:13727021 & + & -40.1 & TACACTAGTTGTGGACATGGAC & - \\
\hline Bro-m003 & A02:16145164:16145426 & + & -54.6 & TCAAAGAATTAGTAGATCGGT & - \\
\hline Bro-m004 & A02:16493715:16493784 & + & -24.9 & - & TCGGACGTTTGTGACGGATTT CC \\
\hline Bro-m005 & A02:21365800:21365890 & + & -22.9 & - & GTGGACACTCTATCTGAGGCT TA \\
\hline Bro-m006 & A02:2629827:2629950 & - & -45.3 & GGAGGCAGCGGTTCATCGATC & - \\
\hline Bro-m007 & A02:22803188:22803422 & - & -39 & - & AGGGATATAGTGTATGGAGTG CA \\
\hline Bro-m008 & A02:25162550:25162723 & - & -32.04 & - & AAGTGGAGTAGAGTATAATGC AG \\
\hline Bro-m009 & A02:25162550:25162724 & + & -47 & AAAGATCTGAACCGAACACGAA & - \\
\hline Bro-m010 & A02:25162550:25162725 & + & -22.1 & TAATCATGTITAGACTTAGATC & - \\
\hline Bro-m011 & A02:25162550:25162726 & - & -21.6 & ACGGTACGGTTITAACAGTTAT & - \\
\hline Bro-m012 & A02:25162550:25162727 & - & -18.5 & AGAGACGATTCTTAACTTTTCTT & - \\
\hline Bro-m013 & A02:25162550:25162728 & - & -51.7 & GATCGAGATCCTTTGGAGTTAGTCC & GCTTGCTAGATGGATCTTGGA CA \\
\hline Bro-m014 & A02:25162550:25162729 & - & -21.9 & - & TGCGGGGTACAAAGACGTTTG \\
\hline Bro-m015 & A02:25162550:25162730 & + & -24.7 & TCTGTCAAGTGGAATGTGGCTT & - \\
\hline Bro-m016 & A02:25162550:25162731 & + & -47.3 & GTTCGGACGCGGCGTCAGACAC & - \\
\hline Bro-m017 & A02:25162550:25162732 & - & -18.1 & TTATTACTTTGTTAGACTCCA & - \\
\hline Bro-m018 & A02:25162550:25162733 & - & -32.4 & TTGACCAGACGACTTATATTTT & - \\
\hline Bro-m019 & A02:25162550:25162734 & - & -51.7 & CGGTCTGTGATGGTACTAGTA & - \\
\hline Bro-m020 & A02:25162550:25162735 & - & -34.7 & TाTGGAGTAATGAGTTGGTTA & - \\
\hline Bro-m021 & A02:25162550:25162736 & + & -104.8 & - & TGGATTGGAACCACATAGGCC \\
\hline Bro-m022 & A02:25162550:25162737 & + & -36.6 & TGGACGACTTAGTAGATGACTT & - \\
\hline Bro-m023 & A02:25162550:25162738 & + & -38 & AATCGATATGGACTAATATGGT & - \\
\hline Bro-m024 & A02:25162550:25162739 & - & -61.7 & TTGCGGTTGCGGACGGTTA & - \\
\hline Bro-m025 & A02:25162550:25162740 & - & -56.1 & TAGCCAAGGATGACTTGCCTG & - \\
\hline Bro-m026 & A02:25162550:25162741 & - & -75.9 & TCTGACTGCTTAGATGATTGCTT & - \\
\hline Bro-m027 & A02:25162550:25162742 & - & -33.9 & - & AGAGACGTCTCTTAGCTTITT TA \\
\hline Bro-m028 & A02:25162550:25162743 & - & -62.3 & - & AGAAGAAACGTAGAAAACAC Tा \\
\hline Bro-m029 & A02:25162550:25162744 & + & -50.5 & CGACAGAAGAGAGTGAGCAC & - \\
\hline Bro-m030 & A02:25162550:25162745 & + & -84.1 & ATATACTCTCAAGCATATCAA & - \\
\hline Bro-m031 & A02:25162550:25162746 & - & -71.8 & - & TTCCCACAAGAACGAAAACTC \\
\hline Bro-m032 & A02:25162550:25162747 & + & -28.4 & AATGGACAAATGGATATGGGCT G & - \\
\hline Bro-m033 & A02:25162550:25162748 & + & -46.3 & TGACAGAAAGAGAGGTGAGCA CG & - \\
\hline Bro-m034 & A02:25162550:25162749 & + & -18.5 & - & TTATTITTGTAGCTATTITTI \\
\hline Bro-m035 & A02:25162550:25162750 & + & -54.2 & TAGCCAAGGATGACTTGCCTG & - \\
\hline Bro-m036 & A02:25162550:25162753 & - & -78.8 & - & TAAGGACCCAAGAGCAAGGA C \\
\hline Bro-m037 & A02:25162550:25162754 & + & -28.3 & CAAGAGATCTGCAGCATGGCGC A & - \\
\hline Bro-m038 & A02:25162550:25162755 & + & -71.1 & TCTTGTTGATTGGTGCATGTCA & - \\
\hline Bro-m039 & A02:25162550:25162756 & - & -105.6 & CAAGCGCTCTTCTCACAGCTT & - \\
\hline Bro-m040 & A02:25162550:25162757 & - & -46 & - & ACGAACCATAGTCCGTCGGTA \\
\hline Bro-m041 & A02:25162550:25162758 & + & -59.2 & - & TTATIIITGTAGCTATIITI A \\
\hline Bro-m042 & A02:25162550:25162760 & - & -22.17 & AAAGGAAAGTTAGAAGACTTCT & - \\
\hline Bro-m043 & A02:25162550:25162761 & - & -50.2 & - & TAAGAAACTTGCAATGGAGGA CA \\
\hline
\end{tabular}




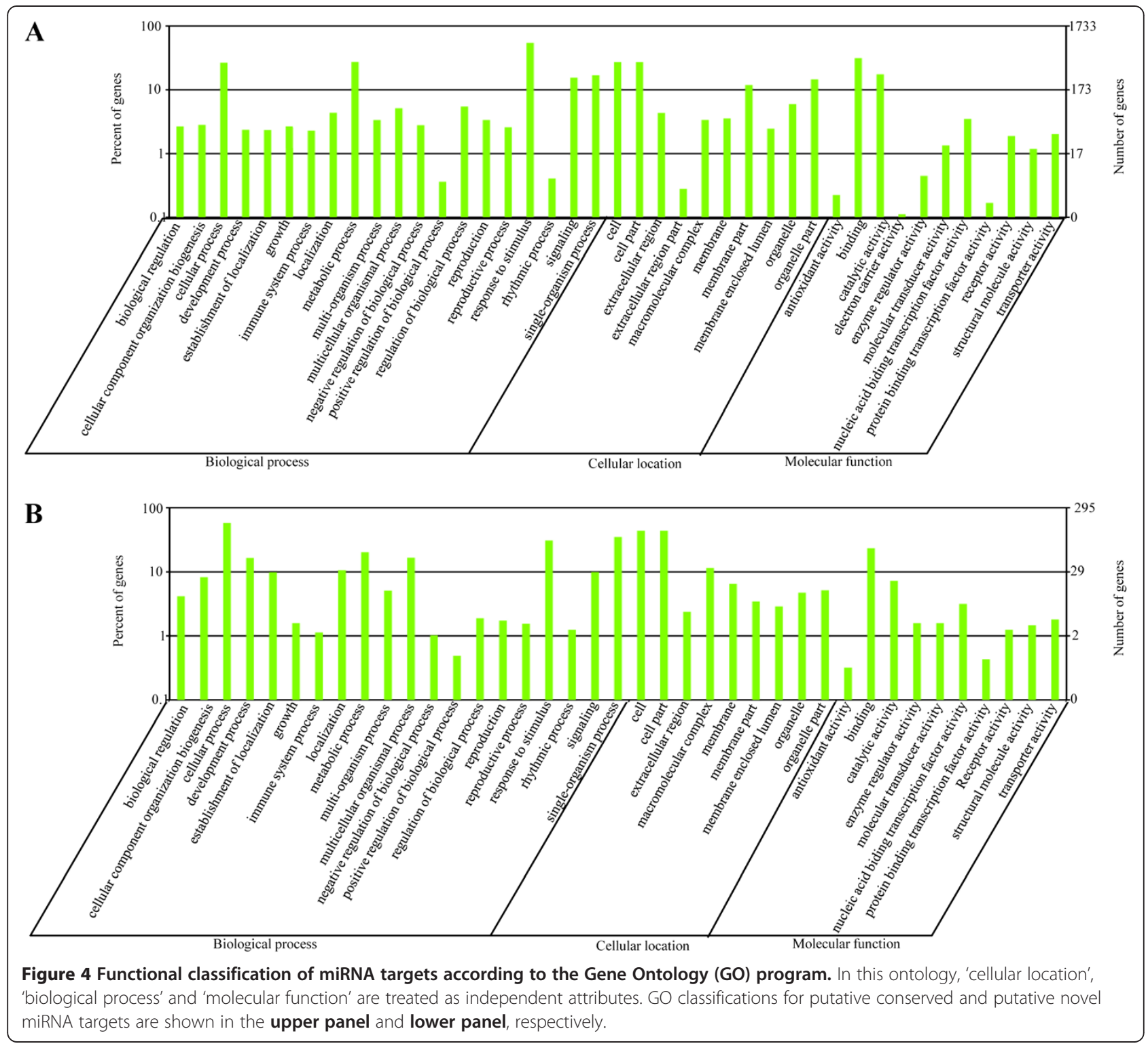

to the identification of miRNAs in broccoli. In the present study, we identified several million sRNA sequences in salt-stressed plantlets of broccoli, using high-throughput sequencing methods to identify miRNAs associated with physiology and metabolism under salt stress. A total of 42 putative known and 39 putative candidate miRNAs that were differentially expressed between control and salt-stressed broccoli were discovered according to their sequencing-reads numbers and confirmed by real-time RT-PCR. Finally, analysis of the predicted targets of the miRNAs using the GO and $\mathrm{KO}$ databases indicated that a range of metabolic pathways and biological processes known to be associated with salt stress were up-regulated in broccoli treated with salt.

The study of miRNAs using traditional methods is complex, and high-throughput sequencing methods now provide a rapid and efficient additional approach by which to identify and profile populations of sRNAs at different stages of plant development. Large numbers of nonconserved or species-specific miRNAs often accumulate in plants at lower levels than conserved miRNAs, and are therefore not easily revealed using traditional sequencing methods $[3,30]$. In the present study, the results indicated a range of sRNAs, of length 16-29 nt, in broccoli, with most of the unique sequence reads being $24 \mathrm{nt}$ in length. Several plant species, including A. thaliana, Citrus sativus and $C$. sinensis, had been shown to contain substantially more 24-nt sRNAs than 21-nt sRNAs [31,32]; on the other hand, sRNAs populations with more 21-nt members than 24-nt were reported in B. juncea and in Japanese apricot with imperfect flower buds [32,33]. B. juncea, B. napus and broccoli all belong to the same genus; however, 
when broccoli sRNAs are compared to those of $B$. juncea, a striking difference exists, whereas little difference is observed when broccoli sRNAs are compared to those of $B$. napus [34]. Thus, we can conclude that the sRNA transcriptome is complex, and can be highly variable even between closely related plant species.

Generally speaking, length distribution analyses of sRNAs provide a helpful way to assess the composition of sRNA samples. Previous studies have shown that DCL1 mainly produces sRNAs that are 18-21 nt long. In contrast, the products of DCL2, DCL3 and DCL4 are $22 \mathrm{nt}, 24 \mathrm{nt}$ and $21 \mathrm{nt}$ long, respectively. Furthermore, miRNAs are normally $21 \mathrm{nt}$ or $22 \mathrm{nt}$ long, whereas small interfering RNA (siRNA) are 24 nt long [35]. By this reasoning, therefore, our results imply that the most abundant sRNAs are miRNAs in broccoli and siRNAs that have been cleaved by DCL1 and DCL4.

Sequence information for broccoli miRNAs is entirely absent from miRBase, and furthermore there are fewer than 50 B. rapa miRNAs in this database. Therefore, the sRNA sequences were aligned instead against miRNA precursors/mature miRNAs of the Viridiplantae in miRBase. A search for conserved miRNAs revealed that the majority of these molecules, known already from A. thaliana and other species, were detectable in broccoli and were expressed at relatively high abundance. The patterns of expression of conserved miRNAs are variable in different plants. Previous studies had shown that miR159, miR166a, miR164, miR171f and miR168 were all detectable in $B$. rapa, and with relatively high numbers of reads, whereas members of the miR169 family showed low read numbers $[30,34]$. The findings of the present study were quite different; miR166a, miR168a and miR157d showed the highest values for copy number in control broccoli.

A previous report suggested that miR403, which was initially identified in A. thaliana and later found in Populus trichocarpa, was a dicot-specific miRNA and that miR437 and miR444 might be monocot-specific miRNAs [36]. On the other hand, Sunkar et al. indicated that miR390 was present in both monocots and dicots [25]. Our results provided a large-scale database against which to examine these suggestions. We detected both miR403 and miR390 in broccoli, whereas miR437 and miR444 were not detectable, thereby confirming the previous reports. Furthermore, the miR158 and miR391 sequences have been considered to be A. thaliana-specific [36]; however, we detected miR158a, miR158b, and miR391 in broccoli, each at an abundance of about 30,000 reads.

Although many plant miRNAs have been found to be conserved - for example, miR319, miR156/157, miR169, miR165/166 and miR394 have been found in more than 40 plant species [36] - studies have indicated that most miRNAs can be induced by environmental stresses. For example, miR399 is induced under low- phosphate conditions [37], miR395 increases upon sulfate starvation [8] and miR393 is up-regulated in response to cold, dehydration, $\mathrm{NaCl}$ and abscisic acid (ABA) stress [38]. A recent study showed that miR417 was transiently up-regulated in response to osmotic stress [39]. On the other hand, miR398 is down-regulated in response to oxidative stress. Several differentially regulated miRNAs have been identified in salt-stressed plants. For example, miR530a, miR1445, miR1446a-e and miR1447 were down-regulated during salt stress in $P$. trichocarpa, as measured by microarray analysis of known miRNAs, whereas miR482.2 and miR1450 were up-regulated [40]. In A. thaliana, miR156, miR158, miR159, miR165, miR167, miR168, miR169, miR171, miR319, miR393, miR394, miR396 and miR397 were all up-regulated in response to salt stress, whereas miR398 was down-regulated. Furthermore, miR169g and miR169n were also reported to be induced by high salinity [41]. Recently, a study of maize roots using miRNA microarray hybridization indicated that members of the miR156, miR164, miR167, and miR396 families were down-regulated by salt shock, whereas miR162 and miR168 were up-regulated [42]. Our result in broccoli indicated that, amongst the conserved miRNAs, miR393 and miR855 were the most strongly down-regulated in response to salt stress, whereas conserved miR838-5p and miR396a were the most strongly up-regulated. Of the putative novel miRNAs, miR37 showed the greatest degree of up-regulation in response to salt stress, whereas miR3 and miR34 showed the greatest degree of down-regulation. The differences between our findings and the reports relating to salt stress in $P$. trichocarpa, A. thaliana and maize roots [41] might be explained in part, of course, by differences in behavior between species but they might also reflect the use of different experiment methods: microarray analysis was used in some of the previous studies, whereas in the present study we used sequencing, which is more effective than microarray analysis for the detection of miRNAs, including novel miRNAs, that are expressed at low levels.

In the case of the human genome, more than a third of the genes have been predicted to be miRNA targets, and these targets would appear to be involved in a wide range of biological functions. In $A$. thaliana, miRNAs may target transcripts that encode proteins and transcription factors [43]. Our results indicated that a total of 836 and 527 mRNAs were targets of putative conserved miRNAs and putative novel miRNAs, respectively. These targets regulate a range of cellular processes, within the broad categories of cell-cycle regulation, plant hormonal signal transduction, and metabolism. However, there are several miRNAs without target genes; these could be the result of erroneous target predictions, or they might be low-abundance miRNAs with limited or no activity. It is also possible that miRNAs might exist that have no 
targets. Nevertheless, the $\mathrm{KO}$ and GO analyses revealed that many of the genes targeted by miRNAs in broccoli are related to salt stress, supporting the hypothesis that miRNAs play an important role in the response of broccoli to salinity.

\section{Conclusion}

A comprehensive study of broccoli miRNAs in relation to salt stress has been undertaken. Our results provide new and significant data on the miRNA profile of broccoli. The differential regulation of miRNAs between control and salt-stressed broccoli indicates that miRNAs have a marked involvement in regulatory networks associated with salt stress. Further studies of gene function and regulation are now required in order to elucidate the precise mechanisms.

\section{Methods}

Plant material and growth conditions

Seeds of broccoli (Brassica oleracea var. italic) were obtained from Sakata Seed Corporation (Yokohama, Kanagawa, Japan). The seeds were cultivated and harvested as previously described [14]. Broccoli is moderately tolerant to salinity $(40-60 \mathrm{mM} \mathrm{NaCl})$, and therefore $80 \mathrm{mM} \mathrm{NaCl}$ was selected in order to study the effects of salt stress. The saline treatment was applied for $15 \mathrm{~d}$, following 1 week of growth. At the end of this period, flowers from control broccoli $(0 \mathrm{mM} \mathrm{NaCl})$ and salt-stressed broccoli were immediately frozen in liquid nitrogen and stored at $-80^{\circ} \mathrm{C}$ before use. In each case, samples were harvested and pooled from four individual flowers.

\section{sRNA library preparation and sequencing}

Total RNA extractions from control broccoli and saltstressed broccoli both of which were pooled from four individual flowers were performed using TRIzol ${ }^{\mathrm{ma}}$ reagent (Invitrogen, Carlsbad, CA, USA), following the manufacturer's instructions. Total RNA quantity and purity were assayed with the NanoDrop ND-2000 spectrophotometer (Thermo Scientific, MA, USA) at 260/280 nm (ratio between 1.8 and 2.0). Moreover, the quality of total RNA was also analyzed by electrophoresis on a denaturing agarose gel and by Agilent Bioanalyzer (Agilent Technologies inc., Santa Clara, CA, USA). The total RNA extractions with RNA integrity number (RIN) value more than 8.0 and without DNA contamination were used for further study. The construction sRNA libraries and deep-sequencing were each performed by the Beijing Genomics Institute (BGI, Shenzhen, China). Briefly, the sRNA fraction, of length 18 to $30 \mathrm{nt}$, was extracted from a $15 \%$ denaturing polyacrylamide gel. The sRNAs were then ligated to a pair of Solexa adapters at their 5'- and 3'- ends, using T4 RNA ligase (New England Biolabs, Ipswich, MA, USA). Next, these sRNAs of length 70 to
$90 \mathrm{nt}$ were extracted from denaturing polyacrylamide gel and converted to DNA by RT-PCR. Finally, the purified PCR products were directly sequenced with a HiSeq 2000 Sequencing System (Illumina, San Diego, CA, USA), used according to the manufacturer's protocol.

\section{sRNA analysis}

The very basic figure from sequencing was converted into sequence data by the base calling step. Such sequence data called raw data or raw reads which was represented by four lines. The second line was the sequence. The fourth line represented the sequencing quality of this read. Each character in this line showed the sequencing quality of the base on the same position in the second line. The actual quality was the corresponding American Standard Code for Information Interchange (ASCII) value of the letter minus 64. The quality of HiSeq sequencing ranged from 0 to 41 . This quality would be used in the criteria for filtering out low quality reads. The sequence tags from the HiSeq sequencing were processed via a data-cleaning pipeline developed by Beijing Genome Institute (BGI, Beijing, China) in order to remove the tags with: any $\mathrm{N}$ bases, more than 4 bases whose quality score was lower than 10 and more than 6 bases whose quality score was lower than 13, and those that were too small (with length shorter than $18 \mathrm{nt}$ ), as well as to remove the adapter sequences from the tags [9]. The remaining sequences generated from Illumina HiSeq and went through data cleaning process were called clean sRNA reads, and they were mapped to the $B$. rapa genome using SOAP to analyze their expression and their distribution within the genome. The program was performed using the following parameters: soap -v 0 -r 2 -m 0 -a clean.fa - d ref_genome. fa.inedx -o match genome.soap. Sequences with perfect match or one mismatch were retained for further analysis. We then used the basic local alignment search tool (BLAST) (http://blast.ncbi.nlm.nih.gov/Blast.cgi) search of sRNAs against the Rfam database in order to remove noncoding RNAs, such as rRNA, tRNA, snRNA and snoRNA. The remaining sequences were then used for further characterization.

\section{Identification of conserved and novel miRNAs}

Following initial processing, homologs of known miRNAs and the remaining non-annotated sRNAs were used to identify miRNAs. Since broccoli miRNA sequences were not available in miRbase, we instead, aligned the sRNA tags against miRNA precursor/mature miRNA sequences of the Viridiplantae belonging to 52 plant species in miRBase (miRBase release 20.0, June, 2013, http://mirbase. org/), in order to identify sequences and miRNA families (not individual species) that were represented in the samples. First, clean data were aligned against the miRNA precursor/mature miRNA sequences, allowing 
two mismatches or gaps to take account of inter-species differences; secondly, for each mature miRNA family, the miRNAs with the highest expression levels were selected as a cluster to create a temporary miRNA database; thirdly, total miRNA expression levels were calculated by summating the clean reads that could align to the temporary miRNA database within two mismatches; finally, sequences identified as miRNAs but which could not fold hairpin structure were categorized as pseudo-miRNAs [44]. Moreover, an adaptive thresholding method using Kolmogorov-Smirnov (KS) statistics was employed to achieve a threshold value, according to the report of Winston et al. [45]. Interestingly, our results indicated that transcripts with a read count of more than 38 are thus deemed to be significantly different from noise both in control and salt-stressed broccoli.

The prediction of novel miRNAs was carried out using a new algorithm termed 'MIREAP', which could identify novel miRNA candidates from their possession of a canonical hairpin structure, and which was adequately supported by sequencing data (http://sourceforge.net/ projects/mireap), developed by the BGI. The following parameters were applied. It includes minimal miRNA length (18), maximal miRNA length (25), minimal miRNA reference sequence length (20), maximal miRNA reference sequence length (23), maximal copy number of miRNAs on reference (20), maximal free energy allowed for a miRNA precursor $(-18 \mathrm{kcal} / \mathrm{mol})$, maximal space (300), minimal mature pair (16), maximal mature bulge (4), maximal duplex asymmetry (4), and flank sequence length (20). Structures that conformed to previously established criteria were considered as candidate novel miRNAs [46]. The stabilities of the candidate pre-miRNAs were assayed using Mfold (http://sourceforge.net/projects/ mireap). Since these miRNAs were mapped to genomic loci on $B$. rapa chromosomes, we denoted then as 'putative novel miRNAs'of broccoli.

\section{Analysis of differential read counts of miRNAs between salt-stressed broccoli and control broccoli}

To compare the differential read counts of miRNAs between salt-stressed broccoli and control broccoli, frequencies of miRNA read counts were normalized as transcripts per million and the normalization of miRNA expression levels between the two broccoli libraries was carried out based on the following formula: normalization of miRNA read counts $(\mathrm{NC})=($ Actual miRNA count / Total count of clean reads $) \times 10^{6}$. If the normalized association value of a given miRNA is zero, its association value was modified to 0.01 ; if the miRNA gene expression of two samples was $<1$, on account of low expression, the miRNA was neglected in the analysis of differential expression. Then, the fold-change and FDR were calculated from the normalized association using the following equations: Fold-change $=\log 2$ (NC of saltstressed broccoli / NC of control broccoli); FDR was calculated using the formula below [32], where: $\mathrm{N}_{1}$ and $\mathrm{N}_{2}$ represent the total counts of clean reads of a given miRNA in the sRNA libraries of control and salt-stressed broccoli samples, respectively; and $x$ and $y$ represent the normalized expression levels of a given miRNA in the sRNA libraries of control and salt-stressed broccoli samples, respectively.

$F D R(x / y)=\left(N_{2} / N_{1}\right)^{y} \frac{(x+y) ! \quad C\left(y \leq y_{\min } x\right)=\sum_{y=0}^{y \leq y_{\min }} F D R(y x)}{x ! y !\left(1+N_{2} / N_{1}\right)^{(x+y+1)}} D\left(y \geq y_{\max } x\right)=\sum_{y \geq y_{\max }}^{\infty} F D R(y x)$

\section{miRNA quantification by real-time RT-PCR}

To validate the results from the bioinformatics-based analysis, stem-loop real-time RT-PCR was performed. Total RNA was first extracted from other three individual control flowers and salt-stressed flowers of broccoli, which were not the same with RNA used for sRNA analysis, using TRIzol ${ }^{\mathrm{TM}}$ reagent. Then, $1 \mu \mathrm{l}$ of the stemloop RT primer $(1 \mu \mathrm{M})$ and $2 \mathrm{mg}$ of total RNA were used in first-strand cDNA synthesis with an iScript ${ }^{\text {Tm }}$ cDNA synthesis kit (Bio-Rad, Hercules, CA, USA). Realtime RT-PCR was carried out using $0.5 \mathrm{ml}$ of cDNA and specifically designed forward primers for each individual miRNA, together with the universal reverse primer, on a Mx3005P qPCR System (Agilent Technologies, Santa Clara, CA, USA), using an $\mathrm{iQ}^{\mathrm{TM}} \mathrm{SYBR}^{\bullet}$ Green Supermix kit (Bio-Rad). The protocol for stem-loop real-time PCR was as follows: initial denaturation at $95^{\circ} \mathrm{C}$ for $10 \mathrm{~s}$, followed by 40 cycles of $10 \mathrm{~s}$ at $95^{\circ} \mathrm{C}$ and $20 \mathrm{~s}$ at $55^{\circ} \mathrm{C}$. At the end of each PCR reaction, a melting curve was determined, and only samples that displayed a one-peak melting curve at the correct annealing temperature were used for subsequent analysis. The threshold was automatically set and the threshold cycle ( $\mathrm{Ct}$ value) was recorded. The real-time RT-PCR reactions were repeated at least three times to ensure statistical rigor. Finally, the miRNA expression was calculated from three independent biological replicates. SnRNA U6 was used as an internal control. Fold changes were calculated by relative quantification. The $2^{\Delta \Delta} C_{\mathrm{T}}$ method has been used to calculate relative changes in gene expression determined from realtime quantitative PCR experiment [47] using the following equations: ${ }^{\Delta} C_{\mathrm{T}}=\left(\mathrm{C}_{\mathrm{T}} \text {, miRNA }-\mathrm{C}_{\mathrm{T}, \mathrm{U} \text {, }}\right)_{\text {Salt-stressed broccoli }}-$ $\left(\mathrm{C}_{\mathrm{T}}\right.$, miRNA $-\mathrm{C}_{\mathrm{T}}$, U6 $)$ Control broccoli, where, the $C_{\mathrm{T}}$ values were directly provided from real-time PCR instrumentation. Statistical differences between groups were assessed using the independent two-sample t-test. Statistical analysis was performed with SPSS statistical package (V15.0). Moreover, the products of PCR were also 
confirmed by electrophoresis sequencing and denaturing gel electrophoresis.

\section{Prediction of miRNA targets}

To explore the biological functions of miRNAs in broccoli, putative target sites were identified by aligning the miRNA sequences to the ESTs of B. rapa, and adopting the alignment criteria suggested by Allen at al. [46] and Schwab et al. [48]. Briefly, the criteria were defined as follows: i) $\leq 4$ mismatches between the miRNA and the target (a G-U base mismatch counts as 0.5 mismatches); ii) no adjacent mismatches in positions $2-12$ of the miRNA/target duplex (counting from the $5^{\prime}$ of the miRNA); iii) $\leq 2$ adjacent mismatches in the miRNA/target duplex; iv) no mismatches in positions $10-11$ and no more than 2.5 mismatches in positions 1-12; and v) the Minimum Free Energy (MFE) of the miRNA/target duplex should be $\geq 75 \%$ of the MFE of the miRNA bound to its perfect complement.

\section{KEGG pathway and GO-enrichment analyses}

In order to investigate further the biological functions of miRNAs and their targets, KEGG pathway analyses were undertaken on the predicted miRNA-target genes. These putative miRNA-target sequences were used as queries against the KEGG database. AGO-enrichment analysis was also undertaken for the target gene candidates. Categories relating to biological processes, cellular components and molecular functions were displayed for the miRNA-target genes. The results revealed those cellular functions that were related significantly to the predicted gene targets of the miRNAs.

\section{Statistical analysis}

Statistical analysis was performed with the SPSS statistical package (v15.0). The results of real-time RT-PCR were repeated three times and data from three independent biological replicates are presented as the mean \pm standard deviation (SD). Statistical differences amongst groups were assessed using the independent two-sample t-test. $P$ values $<0.05$ were considered statistically significant.

\section{Additional files}

Additional file 1: Table S1. Summary of sRNA sequencing data.

Additional file 2: Table S2. Mapping statistics: SRNAs to genome.

Additional file 3: Figure S1. Total and unique clean reads annotated into categories Control broccoli and salt-stressed broccoli are shown in (A) and (B), respectively. Total reads and unique reads are presented in the left panel and right panel, respectively.

Additional file 4: Table S3. The differential expression of putative conserved miRNAs between the two libraries.

Additional file 5: Figure S2. Prediction of the secondary structures of all new candidate miRNAs in salt-stressed broccoli $A$ total of 43 putative novel miRNAs are shown; see also Figure 3B.
Additional file 6: Table S4. Pathway annotations for most of targets of putative conserved miRNAs in salt-stressed broccoli.

Additional file 7: Table S5. Pathway annotations for most of targets of putative novel miRNAs in salt-stressed broccoli.

Competing interests

The authors declare that they have no competing interests.

\section{Authors' contributions}

TY(h) drafted the manuscript and contributed to bioinformatics analyses, manuscript preparation. TY $(\mathrm{m})$ contributed to the miRNA sequencing and qRT-PCR analyses. LX collected plant samples, planned the experimental design and performed the statistical analysis. ZT contributed to isolate the RNA samples and performed critical edition of the manuscript. HZ contributed to miRNA sequencing. LY conceived and organized the experiments. QY contributed to miRNA sequencing. HB collected plant samples and planned the experimental design. SD contributed to bioinformatics analyses and manuscript critical edition. DH collected plant samples and isolated the RNA samples. YK conceived and organized the experiments, and contributed to the miRNA sequencing, the bioinformatics analyses, and the preparation and critiquing of the manuscript. All the authors have read and approved the final manuscript.

\section{Acknowledgements}

The authors would like to thank Guanghui, Xiao, PhD and Dong, Xiao, $\mathrm{PhD}$ for their technical assistance. This study was supported by the National Natural Science Foundation of China-Guangdong Joint Fund (K1060006), the National Science and Technology support program, the Natural Science Foundation of China and the Program for New Century Excellent Talents in Universities.

\section{Author details}

${ }^{1}$ Cancer Research Institute, Southern Medical University, Guangzhou, Guangdong Province, People's Republic of China. ${ }^{2}$ Department of Oncology, Armed Police Hospital of Guangdong Province, Guangzhou, Guangdong Province, People's Republic of China. ${ }^{3}$ State Key Laboratory of Oncology of Southern China, Guangzhou, Guangdong Province, People's Republic of China. ${ }^{4}$ Department of Radiation Oncology, Cancer Center of Sun Yat-Sen University, Guangzhou, Guangdong Province, People's Republic of China.

Received: 25 January 2014 Accepted: 12 August 2014

Published online: 03 September 2014

\section{References}

1. Voinnet O: Origin, biogenesis, and activity of plant microRNAs. Cell 2009, 136(4):669-687.

2. Li A, Mao L: Evolution of plant microRNA gene families. Cell Res 2007, 17(3):212-218

3. Axtell MJ, Jan C, Rajagopalan R, Bartel DP: A two-hit trigger for siRNA biogenesis in plants. Cell 2006, 127(3):565-577.

4. Rogers $\mathrm{K}$, Chen X: Biogenesis, turnover, and mode of action of plant MicroRNAs. Plant Cell 2013, 25(7):2383-2399.

5. Wang L, Liu H, Li D, Chen H: Identification and characterization of maize microRNAs involved in the very early stage of seed germination. BMC Genomics 2011, 12:154.

6. Zhang B, Pan X, Cannon CH, Cobb GP, Anderson TA: Conservation and divergence of plant microRNA genes. Plant J 2006, 46(2):243-259.

7. Sunkar R, Zhou X, Zheng Y, Zhang W, Zhu JK: Identification of novel and candidate miRNAs in rice by high throughput sequencing. BMC Plant Biol 2008, 8:25.

8. Jones-Rhoades MW, Bartel DP: Computational identification of plant microRNAs and their targets, including a stress-induced miRNA. Mol Cell 2004, 14(6):787-799.

9. Liang C, Zhang X, Zou J, Xu D, Su F, Ye N: Identification of miRNA from Porphyra yezoensis by high-throughput sequencing and bioinformatics analysis. PLoS One 2010, 5(5):e10698.

10. Latte KP, Appel KE, Lampen A: Health benefits and possible risks of broccoli - an overview. Food Chem Toxicol 2011, 49(12):3287-3309.

11. Suwabe K, Tsukazaki H, Iketani H, Hatakeyama K, Kondo M, Fujimura M, Nunome T, Fukuoka H, Hirai M, Matsumoto S: Simple sequence repeat- 
based comparative genomics between Brassica rapa and Arabidopsis thaliana: the genetic origin of clubroot resistance. Genetics 2006, 173(1):309-319.

12. Wang $X$, Wang $H$, Wang J, Sun $R$, Wu J, Liu S, Bai $Y$, Mun JH, Bancroft I, Cheng F, Huang S, Li X, Hua W, Wang J, Wang X, Freeling M, Pires JC, Paterson AH, Chalhoub B, Wang B, Hayward A, Sharpe AG, Park BS, Weisshaar B, Liu B, Li B, Liu B, Tong C, Song C, Duran C, et al: The genome of the mesopolyploid crop species Brassica rapa. Nat Genet 2011, 43(10):1035-1039.

13. Geng Y, Wu R, Wee CW, Xie F, Wei X, Chan PM, Tham C, Duan L, Dinneny JR: A spatio-temporal understanding of growth regulation during the salt stress response in Arabidopsis. Plant Cell 2013, 25(6):2132-2154.

14. Muries B, Carvajal M, Martinez-Ballesta Mdel C: Response of three broccoli cultivars to salt stress, in relation to water status and expression of two leaf aquaporins. Planta 2013, 237(5):1297-1310.

15. Jian X, Zhang L, Li G, Zhang L, Wang X, Cao X, Fang X, Chen F: Identification of novel stress-regulated microRNAs from Oryza sativa $L$. Genomics 2010, 95(1):47-55.

16. Martin RC, Liu PP, Goloviznina NA, Nonogaki H: microRNA, seeds, and Darwin?: diverse function of miRNA in seed biology and plant responses to stress. J Exp Bot 2010, 61(9):2229-2234.

17. Carnavale Bottino M, Rosario S, Grativol C, Thiebaut F, Rojas CA, Farrineli L, Hemerly AS, Ferreira PC: High-throughput sequencing of small RNA transcriptome reveals salt stress regulated microRNAs in sugarcane. PLoS One 2013, 8(3):e59423.

18. Li B, Duan H, Li J, Deng XW, Yin W, Xia X: Global identification of miRNAs and targets in Populus euphratica under salt stress. Plant Mol Biol 2013, 81(6):525-539.

19. De Paola D, Cattonaro F, Pignone D, Sonnante G: The miRNAome of globe artichoke: conserved and novel micro RNAs and target analysis. BMC Genomics 2012, 13:41.

20. Li R, Yu C, Li Y, Lam TW, Yiu SM, Kristiansen K, Wang J: SOAP2: an improved ultrafast tool for short read alignment. Bioinformatics 2009, 25(15):1966-1967.

21. Chorostecki U, Crosa VA, Lodeyro AF, Bologna NG, Martin AP, Carrillo N, Schommer C, Palatnik JF: Identification of new microRNA-regulated genes by conserved targeting in plant species. Nucleic Acids Res 2012, 40(18):8893-8904.

22. Varkonyi-Gasic E, Wu R, Wood M, Walton EF, Hellens RP: Protocol: a highly sensitive RT-PCR method for detection and quantification of microRNAs. Plant Methods 2007, 3:12

23. Chen C, Ridzon DA, Broomer AJ, Zhou Z, Lee DH, Nguyen JT, Barbisin M, Xu NL, Mahuvakar VR, Andersen MR, Lao KQ, Livak KJ, Guegler KJ: Real-time quantification of microRNAs by stem-loop RT-PCR. Nucleic Acids Res 2005, 33(20):e179.

24. Allen E, Xie Z, Gustafson AM, Sung GH, Spatafora JW, Carrington JC: Evolution of microRNA genes by inverted duplication of target gene sequences in Arabidopsis thaliana. Nat Genet 2004, 36(12):1282-1290.

25. Sunkar R, Girke T, Zhu JK: Identification and characterization of endogenous small interfering RNAs from rice. Nucleic Acids Res 2005, 33(14):4443-4454.

26. Burssens $S$, Himanen $K$, van de Cotte B, Beeckman T, Van Montagu M, Inze $D$, Verbruggen $N$ : Expression of cell cycle regulatory genes and morphological alterations in response to salt stress in Arabidopsis thaliana. Planta 2000, 211(5):632-640

27. Xiong L, Schumaker KS, Zhu JK: Cell signaling during cold, drought, and salt stress. Plant Cell 2002, 14(Suppl):S165-S183.

28. Tsuzuki M, Moskvin OV, Kuribayashi M, Sato K, Retamal S, Abo M, Zeilstra-Ryalls J, Gomelsky M: Salt stress-induced changes in the transcriptome, compatible solutes, and membrane lipids in the facultatively phototrophic bacterium Rhodobacter sphaeroides. Appl Environ Microbiol 2011, 77(21):7551-7559.

29. Zhu JK: Regulation of ion homeostasis under salt stress. Curr Opin Plant Biol 2003, 6(5):441-445

30. Fahlgren N, Howell MD, Kasschau KD, Chapman EJ, Sullivan CM, Cumbie JS, Givan SA, Law TF, Grant SR, Dangl JL, Carrington JC: High-throughput sequencing of Arabidopsis microRNAs: evidence for frequent birth and death of MIRNA genes. PLoS One 2007, 2(2):e219.

31. Rajagopalan R, Vaucheret $H$, Trejo J, Bartel DP: A diverse and evolutionarily fluid set of microRNAs in Arabidopsis thaliana. Genes Dev 2006, 20(24):3407-3425.
32. Gao Z, Shi T, Luo X, Zhang Z, Zhuang W, Wang L: High-throughput sequencing of small RNAs and analysis of differentially expressed microRNAs associated with pistil development in Japanese apricot. BMC Genomics 2012, 13:371.

33. Yang J, Liu X, Xu B, Zhao N, Yang X, Zhang M: Identification of miRNAs and their targets using high-throughput sequencing and degradome analysis in cytoplasmic male-sterile and its maintainer fertile lines of Brassica juncea. BMC Genomics 2013, 14:9.

34. Xu MY, Dong Y, Zhang QX, Zhang L, Luo YZ, Sun J, Fan YL, Wang L: Identification of miRNAs and their targets from Brassica napus by high-throughput sequencing and degradome analysis. BMC Genomics 2012, 13:421.

35. Bartel DP: MicroRNAs: genomics, biogenesis, mechanism, and function. Cell 2004, 116(2):281-297

36. Sunkar $R$, Jagadeeswaran G: In silico identification of conserved microRNAs in large number of diverse plant species. BMC Plant Biol 2008, 8:37.

37. Fujii H, Chiou TJ, Lin Sl, Aung K, Zhu JK: A miRNA involved in phosphatestarvation response in Arabidopsis. Curr Biol 2005, 15(22):2038-2043.

38. Sunkar R, Chinnusamy V, Zhu J, Zhu JK: Small RNAs as big players in plant abiotic stress responses and nutrient deprivation. Trends Plant Sci 2007, 12(7):301-309.

39. Shukla LI, Chinnusamy V, Sunkar R: The role of microRNAs and other endogenous small RNAs in plant stress responses. Biochim Biophys Acta 2008, 1779(11):743-748.

40. Lu S, Sun YH, Chiang VL: Stress-responsive microRNAs in Populus. Plant J 2008, 55(1):131-151.

41. Khraiwesh B, Zhu JK, Zhu J: Role of miRNAs and siRNAs in biotic and abiotic stress responses of plants. Biochim Biophys Acta 2012, 1819(2):137-148.

42. Ding D, Zhang L, Wang H, Liu Z, Zhang Z, Zheng Y: Differential expression of miRNAs in response to salt stress in maize roots. Ann Bot 2009, 103(1):29-38.

43. Sunkar R, Girke T, Jain PK, Zhu JK: Cloning and characterization of microRNAs from rice. Plant Cell 2005, 17(5):1397-1411.

44. Ruby JG, Jan C, Player C, Axtell MJ, Lee W, Nusbaum C, Ge H, Bartel DP: Large-scale sequencing reveals 21U-RNAs and additional microRNAs and endogenous siRNAs in C. elegans. Cell 2006, 127(6):1193-1207.

45. Koh W, Sheng CT, Tan B, Lee QY, Kuznetsov V, Kiang LS, Tanavde V: Analysis of deep sequencing microRNA expression profile from human embryonic stem cells derived mesenchymal stem cells reveals possible role of let-7 microRNA family in downstream targeting of hepatic nuclear factor 4 alpha. BMC Genomics 2010, 11(Suppl 1):S6.

46. Allen E, Xie Z, Gustafson AM, Carrington JC: microRNA-directed phasing during trans-acting siRNA biogenesis in plants. Cell 2005, 121(2):207-221.

47. Livak KJ, Schmittgen TD: Analysis of relative gene expression data using real-time quantitative PCR and the 2(-Delta Delta C(T)) Method. Methods 2001, 25(4):402-408.

48. Schwab R, Palatnik JF, Riester M, Schommer C, Schmid M, Weigel D: Specific effects of microRNAs on the plant transcriptome. Dev Cell 2005, 8(4):517-527.

doi:10.1186/s12870-014-0226-2

Cite this article as: Tian et al:: Identification and characterization of microRNAs related to salt stress in broccoli, using high-throughput sequencing and bioinformatics analysis. BMC Plant Biology 2014 14:226.

\section{Submit your next manuscript to BioMed Central and take full advantage of:}

- Convenient online submission

- Thorough peer review

- No space constraints or color figure charges

- Immediate publication on acceptance

- Inclusion in PubMed, CAS, Scopus and Google Scholar

- Research which is freely available for redistribution 